\title{
Um Estudo da Informatização em Empresas Industriais Paulistas
}

\author{
Antonio Geraldo da Rocha Vidal \\ Ronaldo Zwicker \\ César Alexandre de Souza
}

\begin{abstract}
Resumo
Este trabalho tem como objetivo o estudo dos principais recursos envolvidos no uso de Tecnologia da Informação por empresas industriais, relacionados com a infra-estrutura de tecnologia, sistemas de informação, negócios eletrônicos, recursos humanos e investimentos. Inicialmente são discutidos conceitos relacionados ao assunto e em seguida são apresentados os resultados de uma pesquisa de natureza descritiva, conduzida em 326 indústrias paulistas de pequeno, médio e grande porte. Os resultados permitem caracterizar o emprego de recursos de TI e estabelecer comparações entre os diferentes portes de empresa em relação às variáveis pesquisadas. Este trabalho representa a primeira parte de um projeto de especificação de métricas e instrumentos que possibilitem avaliar o nível de informatização de empresas industriais.
\end{abstract}

Palavras-chave: gestão de tecnologia da informação; informatização; uso organizacional da TI; pequenas e médias empresas.

\section{Abstract}

The objective of this paper is the study of the main aspects of the Informatization process in industrial companies related to technology infrastructure, information systems (IS) use, information technology (IT) management and e-business. Initially are discussed concepts related to the subject and next are presented the results of an exploratory survey carried out in 326 small, medium and large Brazilian industries. Results characterize the main IT resources utilized and set up comparisons among the different company sizes with regard to the surveyed variables. This work constitutes the first part of a project that aims the development of measuring specifications and instruments that will assess the level of IT use in private companies.

Key words: IT Management; Informatization; Organizational Use of IT; Small and Medium Business 


\section{INTRODUÇÃO}

O objetivo deste trabalho é estudar os principais recursos envolvidos no uso de Tecnologia da Informação (TI) por empresas industriais. A importância do nível de informatização de empresas privadas fica evidente, quando se considera a grande diferença no uso da TI entre os países desenvolvidos e os chamados países emergentes, como é o caso do Brasil. O trabalho apresenta os resultados de uma pesquisa descritiva realizada em 326 indústrias paulistas, divididas entre pequenas (149), médias (99) e grandes empresas (78). Diversas variáveis foram analisadas visando a comparar estes grupos de empresas. Maior ênfase foi dada à análise do nível de informatização das pequenas e médias empresas com objetivo de identificar diferenças e características em relação às empresas de grande porte.

O trabalho visa a validar os argumentos apresentados no item Modelo para Avaliação da Informatização de Organizações, o que lhe confere natureza exploratória. A partir da validação da proposta e do desenvolvimento de uma metodologia de avaliação adequada ao contexto empresarial nacional, imagina-se ser possível obter recomendações que possam contribuir para fomentar a atividade empresarial. Este trabalho constitui a etapa inicial de um projeto de especificação de métricas e instrumentos para a avaliação do nível de informatização de empresas de acordo com seu porte e setor de atividade.

\section{A Informatização e suas Dimensões}

Diversos governos de países em desenvolvimento têm mostrado preocupação com a questão da informatização de seus órgãos, empresas e da sociedade como um todo (AZAD; ERDEM; SALEEM, 1998; LIM, 2001). Para esses autores, a competitividade de seus países e empresas no mercado global depende, entre outros fatores, do alcance de determinados níveis ou graus de informatização que devem ser atingidos por suas sociedade e indústrias. Para isso hão de contribuir as políticas públicas e a infra-estrutura de telecomunicações. Outra importante questão é a educação, que é necessária para formar os trabalhadores do conhecimento que irão desenvolver e utilizar os sistemas e tecnologias da informação, como forma de atingir a competitividade empresarial e o desenvolvimento socioeconômico. 
Nesse contexto a Agência Nacional de Informatização da Coréia do Sul (apud LIM, 2001, p. 144) define informatização como "a conversão dos principais recursos e energias de uma economia social para informações, por meio da revolução gerada pela tecnologia de comunicação de dados, e utilizando as informações produzidas mediante a consolidação, processamento e distribuição de dados dentro dos vastos campos da sociedade”. Nessa definição é implícita a importância da utilização generalizada de tecnologias da informação em toda a sociedade. No Brasil a preocupação com esse aspecto é evidenciada pelo Livro Verde, publicado pela Sociedade da Informação do Brasil (TAKAHASHI, 2000). Os seus autores também consideram a informatização das organizações como um dos importantes pilares da informatização da sociedade.

O termo informatização pode ser associado ao conceito de empresa digital e que significa a maximização do uso da TI por uma empresa para a realização de seus negócios. Laudon e Laudon (2001, p. 6) definem a empresa digital como aquela onde "praticamente todos os processos de negócio e relacionamentos com parceiros, clientes e funcionários são viabilizados por meios digitais e recursos chaves corporativos são gerenciados também por meios digitais".

Tu (2001) define o uso organizacional da tecnologia de informação como "a extensão com que uma organização utiliza sistemas de informação para promover a integração e oferecer suporte à decisão e ao seu planejamento estratégico". Para o autor, as quatro principais dimensões do uso organizacional dos sistemas de informação (SI) são:

. Suporte das decisões operacionais ou uso dos SI para monitorar, coordenar e melhorar os processos de decisão ligados às atividades operacionais.

. Suporte do planejamento estratégico ou uso dos SI para formular, coordenar e melhorar os processos de planejamento de longo prazo.

- Integração interna ou uso dos SI para facilitar a troca de informações e a coordenação das atividades dentro da organização.

. Integração externa ou uso dos SI para a comunicação com elementos externos à organização, tais como clientes, fornecedores, bancos etc.

Doll e Torkzadeh (1998) também apresentam dimensões semelhantes para o uso organizacional dos sistemas de informação, dividindo-as em três categorias: suporte da decisão, que inclui o uso para resolução de problemas e apoio na tomada de decisões; integração do trabalho, que inclui a integração horizontal 
e vertical e; serviço ao cliente, extensão em que os SI são utilizados para servir as pessoas, pertençam elas ou não à organização.

Já Lucas (1997) apresenta o conceito de Technology-Form Organization. A T-Form Organization é uma organização que emprega a tecnologia da informação para se tornar altamente eficiente e eficaz. O uso intensivo de TI nessas organizações evidencia as seguintes características: estrutura organizacional achatada, por conta do uso intensivo de ferramentas de apoio a grupos de trabalho; alto nível de delegação de tarefas e de confiança entre subordinados e gerentes; infra-estrutura composta por redes e computadores conectados interna e externamente; gerenciamento de TI forte e capacitado; formação de forças-tarefas temporárias para projetos que envolvem elementos internos da organização, fornecedores e clientes com uso de trabalho remoto. A definição está focada nos resultados do uso da TI e aponta a necessidade da adequação da infra-estrutura (de hardware e comunicações) e para o uso dos SI para integração horizontal e externa. Também está implícito o argumento de que o uso da TI transforma as organizações.

\section{Modelo para Avaliação da Informatização de Organizações}

Neste trabalho a informatização corresponde ao uso gerenciado de recursos de TI para apoiar e desenvolver uma organização visando otimizar seu desempenho. As dimensões que serão consideradas são: (1) infra-estrutura, relacionada aos recursos de tecnologia de hardware, software básico e comunicações; (2) uso, relacionada aos recursos e processos baseados em sistemas de informações e; (3) gestão, relacionada aos recursos humanos e investimentos de TI. A dimensão uso contempla a integração horizontal entre processos e atividades da cadeia de valor; a integração vertical entre os níveis hierárquicos; a integração externa com parceiros, clientes e fornecedores; o suporte da decisão e a obtenção de vantagem competitiva, baseada no uso inovador de TI. A Figura 1 sugere o arcabouço estrutural do modelo de pesquisa adotado. 


\section{Figura 1: Modelo de Pesquisa}

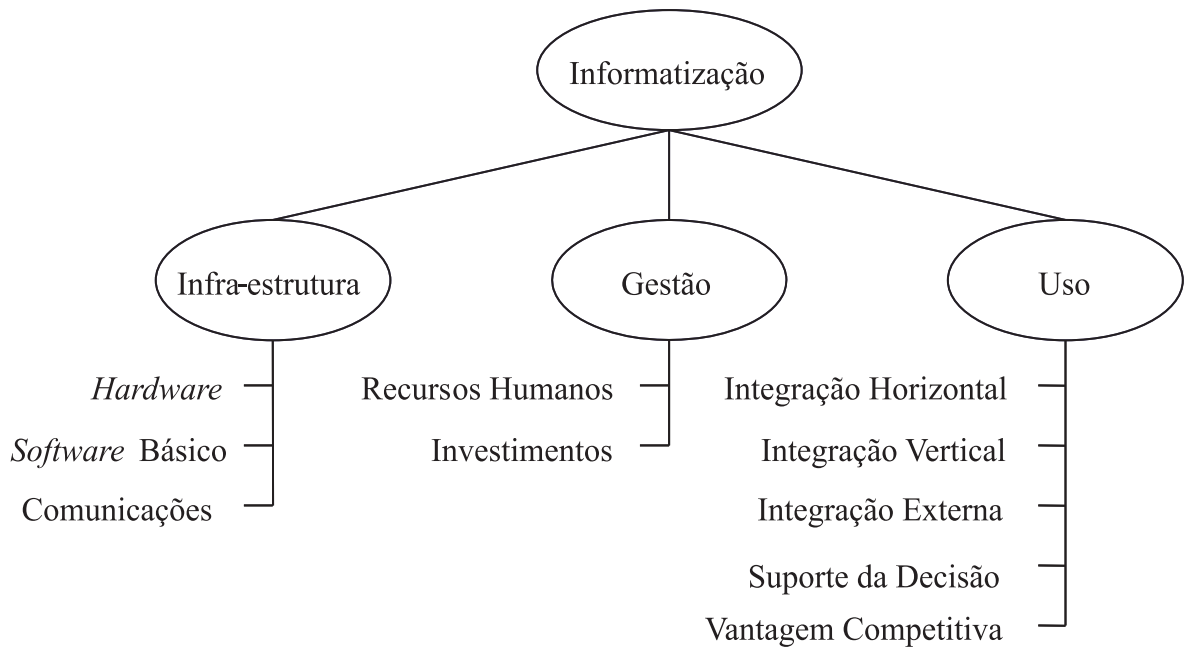

O modelo se baseia nos recursos típicos de TI disponíveis nas empresas para analisar a sua informatização. São recursos de natureza concreta e que, em princípio, podem ser imediatamente contabilizados. Para avaliar os reflexos do uso desses recursos também foram pesquisados os impactos da TI na empresa e que foram mensurados subjetivamente pelos respondentes. Em síntese o objetivo do modelo é permitir obter uma visão inicial e parcial do nível de informatização das empresas industriais paulistas.

Conforme Prenkumar (2003), na gestão de tecnologia da informação comparecem diversos aspectos que podem influir decisivamente no processo de informatização de uma empresa. Podem ser considerados aspectos importantes no âmbito da TI: o nível de centralização das decisões, o grau de formalização do ambiente, o patrocínio de empreendimentos, a experiência da área, o perfil do executivo, os recursos disponíveis, o suporte de sistemas de informações e o grau de terceirização. Na gestão de TI também comparecem aspectos de vantagem competitiva, planejamento e organização que mantêm relação direta com a estratégia e estrutura da empresa.

Essa multiplicidade de aspectos não é considerada no modelo proposto; além disso, o modelo também não captura a influência do ambiente externo e da empresa no processo de informatização. Claramente o uso de determinadas tecnologias e a implementação de determinados processos podem depender de decisões de pessoas da empresa não envolvidas com a TI, como também podem depender de decisões condicionadas a fatores externos e alheios à própria organização; portanto a dimensão gestão que 
comparece no modelo não deve ser interpretada no sentido amplo da gestão de TI.

Finalmente cabe assinalar que, segundo a definição adotada, a informatização deve ser conduzida na organização visando a melhorar seu desempenho. Esse pressuposto é controverso, uma vez que ainda não foram estabelecidas relações causais seguras entre o grau de informatização e o desempenho de uma organização. Na pesquisa realizada, as empresas foram solicitadas a avaliar os impactos do uso da TI sobre alguns aspectos de seu desempenho; entretanto, considerando a proposta do estudo, essa discussão não será aprofundada neste trabalho.

\section{Metodologia}

A pesquisa foi realizada em 2002 em parceria com a Federação das Indústrias do Estado de São Paulo (FIESP). Ela envolveu a coleta de dados por meio de questionários enviados a uma amostra de 3.000 empresas constantes do cadastro da FIESP com retorno de 383 dos quais 326 foram considerados adequados para este estudo (11\% dos questionários enviados). A coleta de dados ocorreu durante os meses de novembro e dezembro e se deu por meio do envio de versão impressa do questionário e senha de acesso para a correspondente versão Internet a cada uma das empresas selecionadas.

A amostra, selecionada por conveniência, atendeu às seguintes premissas: (a) o total de empresas foi definido pela FIESP como sendo 3.000; (b) foram incluídas todas as 534 empresas de grande porte que fazem parte do cadastro da FIESP; (c) 50\% das empresas da amostra deveriam ser de grande e médio porte e 50\% de pequeno porte e; (d) foram incluídas na amostra as 1.781 empresas que já haviam participado de outra pesquisa similar, realizada pela FIESP em 2000 (FIESP/FIPE, 2000). O restante da amostra foi obtido por sorteio com base no cadastro completo da FIESP.

O cadastro da FIESP contava com 13.977 empresas, divididas em 534 grandes (3,8\%), 2.833 médias (20,3\%), 9.497 pequenas (67,9\%) e 1.113 micro (8,0\%). O objetivo da divisão da amostra em $50 \%$ de pequenas e $50 \%$ de médias e grandes era a obtenção de um número significativo de empresas médias e grandes que permitisse a comparação entre os três grupos. Assim, embora os resultados gerais não possam ser extrapolados diretamente à população (cadastro FIESP), eles podem ser analisados e extrapolados por porte. Para a divisão das empresas 
respondentes por porte foi utilizado o critério da FIESP: empresas de 1 a 9 funcionários são consideradas microempresas; de 10 a 99 são empresas pequenas; de 100 a 499 são empresas médias e acima de 500 são empresas grandes. As empresas que não informaram o número de funcionários na pesquisa foram classificadas de acordo com a informação existente no cadastro da FIESP. Nas análises relatadas neste trabalho foram consideradas como pequenas as empresas de 0 a 99 funcionários.

O questionário visava avaliar o grau de utilização da TI na gestão interna e nos negócios da empresa de acordo com as dimensões propostas: infraestrutura, uso e gestão. No texto do trabalho a dimensão uso foi analisada segundo os sistemas de informações e negócios eletrônicos da empresa; e a dimensão gestão foi analisada segundo as atividades, recursos humanos e investimentos da área de TI. Os resultados são apresentados segundo as dimensões e permitem caracterizar o emprego de recursos de TI pelas empresas pesquisadas, bem como sua aplicação e impacto nos negócios. As análises estatísticas foram realizadas com o auxílio do software SPSS for Windows versão 11.0 .

\section{AnÁlise dos Dados}

\section{Descrição da Amostra}

A Tabela 1 apresenta o número de empresas da amostra por porte. A distribuição obtida corresponde à amostra para envio do questionário. A Tabela 2 apresenta a origem do capital das empresas. As empresas também informaram a sua atividade econômica por meio do Código Nacional de Atividade Econômica (CNAE). A Tabela 3 sumariza estes resultados.

\section{Tabela 1: Características da Amostra}

\begin{tabular}{|c|c|c|c|c|c|c|c|c|c|c|c|}
\hline \multirow[t]{2}{*}{ Porte } & \multicolumn{2}{|c|}{ Amostra } & \multicolumn{3}{|c|}{$\begin{array}{c}\text { Faturamento Médio } \\
(R \$ \text { mil })\end{array}$} & \multicolumn{3}{|c|}{$\begin{array}{c}\text { Total de } \\
\text { Funcionários }\end{array}$} & \multicolumn{3}{|c|}{$\begin{array}{c}\text { Funcionários na } \\
\text { Produção (\%) }\end{array}$} \\
\hline & $n$ & $\%$ & média & $d p$ & $n$ & média & $d p$ & $n$ & média & $d p$ & $n$ \\
\hline Pequenas & 149 & 45,7 & 5.492 & 13.302 & 122 & 45 & 26 & 139 & $64 \%$ & $19 \%$ & 138 \\
\hline Médias & 99 & 30,4 & 46.541 & 67.478 & 78 & 226 & 102 & 87 & $76 \%$ & $16 \%$ & 84 \\
\hline Grandes & 78 & 23,9 & 420.801 & 544.794 & 57 & 1.998 & 1.912 & 63 & $76 \%$ & $20 \%$ & 55 \\
\hline Total & 326 & 100,0 & & & & & & & & & \\
\hline
\end{tabular}


Tabela 2: Origem do Capital

\begin{tabular}{|l|c|c|c|}
\hline \multicolumn{1}{|c|}{ Natureza } & Pequena & Média & Grande \\
\hline Capital Privado Nacional & $91 \%$ & $70 \%$ & $51 \%$ \\
\hline Capital Privado Estrangeiro & $5 \%$ & $10 \%$ & $32 \%$ \\
\hline Capital Privado Misto & $2 \%$ & $9 \%$ & $5 \%$ \\
\hline Outros & $1 \%$ & $2 \%$ & $5 \%$ \\
\hline Não informado & $1 \%$ & $8 \%$ & $6 \%$ \\
\hline
\end{tabular}

Tabela 3: Atividade Econômica

\begin{tabular}{|c|c|c|c|c|}
\hline Atividade Econômica & Total & Pequena & Média & Grande \\
\hline Fabricação de máquinas e equipamentos & $16 \%$ & $19 \%$ & $15 \%$ & $10 \%$ \\
\hline Fabricação de produtos de metal (exclusive máquinas e equipamentos) & $11 \%$ & $14 \%$ & $10 \%$ & $8 \%$ \\
\hline Fabricação de produtos químicos & $10 \%$ & $9 \%$ & $9 \%$ & $13 \%$ \\
\hline Fabricação de bebidas e produtos alimentícios & $9 \%$ & $7 \%$ & $11 \%$ & $10 \%$ \\
\hline Fabricação de artigos de borracha e plástico & $8 \%$ & $7 \%$ & $9 \%$ & $8 \%$ \\
\hline Fabricação e montagem de veículos automotores e complementos & $6 \%$ & $5 \%$ & $2 \%$ & $10 \%$ \\
\hline Demais códigos CNAE & $41 \%$ & $39 \%$ & $43 \%$ & $41 \%$ \\
\hline
\end{tabular}

\section{Dimensão Infra-estrutura de Tecnologia}

A Tabela 4 apresenta as descrições e estatísticas das variáveis relacionadas à infra-estrutura de tecnologia. Consta a média (M), o desvio padrão (S) e a quantidade de casos válidos (n) da amostra para cada variável em cada um dos grupos de empresas. Também é informado o coeficiente de assimetria (CA), calculado com base na medida de skewness fornecida pelo SPSS, dividida pelo seu desvio padrão. Consideram-se simétricas distribuições em que este valor se situa na faixa entre -1,96 e 1,96.

Para verificar se há diferença estatisticamente significativa em relação aos três grupos de empresas utilizou-se o teste ANOVA. Segundo Glass e Hopkins (1996), o teste ANOVA é robusto para distribuições não normais; mas, quando a comparação se dá entre grupos com diferentes variâncias, o teste pode não fornecer resultados precisos. Nesses casos, pode-se utilizar o teste de BrownForsythe (GLASS; HOPKINS, 1996, p. 405) que não assume o pressuposto da homogeneidade da variância e fornece um resultado corrigido para o coeficiente $F\left(F^{*}\right)$. Para verificar a hipótese nula de homogeneidade entre as variâncias dos grupos, pode-se utilizar o teste de Levene. Após a rejeição da hipótese nula de igualdade entre os grupos, fornecida pela ANOVA, é necessário proceder à realização de testes post-hoc, que realizam a comparação dos grupos em pares para a determinação de grupos que diferem entre si. 
Tabela 4: Variáveis de Infra-estrutura de Tecnologia

\begin{tabular}{|c|c|c|c|c|c|c|c|c|c|c|c|c|c|}
\hline \multirow{2}{*}{ Variável } & \multirow{2}{*}{ Descrição } & \multicolumn{4}{|c|}{ Pequena } & \multicolumn{4}{|c|}{ Média } & \multicolumn{4}{|c|}{ Grande } \\
\hline & & $M$ & $S$ & $C A$ & $n$ & $M$ & $S$ & $C A$ & $n$ & $M$ & $S$ & $C A$ & $n$ \\
\hline ITEQP & $\begin{array}{c}\text { Total de equipamentos } \\
(\text { PCs }+ \text { notebooks }+ \text { palmtops })\end{array}$ & 12,8 & 12,2 & 14,9 & 149 & 57,8 & 50,3 & 8,3 & 99 & 786,8 & 1481 & 17,9 & 78 \\
\hline ITEQPFUN & $\begin{array}{l}\text { Equipamentos por } \\
\text { funcionário }\end{array}$ & 0,32 & 0,24 & 9,8 & 139 & 0,25 & 0,19 & 6,7 & 87 & 0,30 & 0,2 & 3,4 & 63 \\
\hline ITEQPADM & $\begin{array}{c}\text { Equipamentos por funcionário } \\
\text { exceto produção }\end{array}$ & 0,97 & 0,66 & 8,6 & 137 & 1,26 & 0,89 & 6,5 & 84 & 1,38 & 0,9 & 3,5 & 55 \\
\hline ITMOV & $\begin{array}{l}\% \text { de equipamentos móveis } \\
\text { (notebooks + palmtops) }\end{array}$ & $7 \%$ & $11 \%$ & 11,3 & 149 & $7 \%$ & $9 \%$ & 12,3 & 98 & $12 \%$ & $14 \%$ & 7,3 & 78 \\
\hline ITMOVCOM & $\begin{array}{l}\text { Equipamentos móveis por } \\
\text { funcionário da área comercial }\end{array}$ & 0,18 & 0,30 & 8,0 & 133 & 0,35 & 0,57 & 12,6 & 78 & 0,88 & 1,1 & 7,2 & 51 \\
\hline ITRED & $\begin{array}{l}\% \text { de equipamentos } \\
\text { conectados em rede }\end{array}$ & $71 \%$ & $39 \%$ & $-5,4$ & 149 & $92 \%$ & $12 \%$ & $-9,0$ & 90 & $91 \%$ & $15 \%$ & $-7,5$ & 67 \\
\hline ITINT & $\begin{array}{l}\% \text { de equipamentos } \\
\text { conectados à Internet }\end{array}$ & $66 \%$ & $30 \%$ & $-1,2$ & 132 & $62 \%$ & $31 \%$ & $-0,7$ & 88 & $53 \%$ & $30 \%$ & 1,1 & 67 \\
\hline ITTEMRED & $\begin{array}{c}\% \text { de empresas } \\
\text { que possuem rede }\end{array}$ & $62 \%$ & & & 149 & $97 \%$ & & & 99 & $100 \%$ & & & 78 \\
\hline ITSEREQP & $\begin{array}{l}\text { Número de servidores por } \\
\text { total de equipamentos }\end{array}$ & 0,08 & 0,09 & 11,6 & 145 & 0,06 & 0,04 & 4,7 & 98 & 0,04 & 0,0 & 9,9 & 78 \\
\hline
\end{tabular}

A Tabela 5 apresenta as significâncias dos testes de Levene, da ANOVA e do teste de Brown-Forsythe pertinentes a variáveis para as quais a hipótese de variâncias homogêneas entre os grupos foi rejeitada. Também são apresentadas as significâncias do teste post-hoc de Tukey-Kramer, adequado quando os grupos têm tamanhos diferentes e a variância é homogênea entre os grupos, ou do teste de Games-Howell, adequado às variâncias não homogêneas (GARSON, 2003).

\section{Tabela 5: Testes de Significância das Variáveis de Infra-estrutura de Tecnologia}

\begin{tabular}{|c|c|c|c|c|c|c|c|c|}
\hline \multirow{2}{*}{ Variável } & \multirow{2}{*}{$\begin{array}{l}\text { Teste de } \\
\text { Levene }\end{array}$} & \multicolumn{2}{|c|}{ ANOVA } & \multicolumn{2}{|c|}{ Brown-Forsythe } & \multicolumn{3}{|c|}{ Games-Howell ou Tukey-Kramer } \\
\hline & & $F$ & Sig. & $F^{*}$ & Sig. & $P Q$ vs $M D$ & $P Q v s G D$ & $M D$ vs $G D$ \\
\hline ITEQPFUN & 0,06 & 1,97 & 0,14 & --- & --- & --- & --- & --- \\
\hline ITEQPADM & 0,01 & 6,71 & 0,00 & 5,94 & $0,00 *$ & $0,03 *$ & $0,01 *$ & 0,69 \\
\hline ITMOV & 0,01 & 5,68 & 0,00 & 5,42 & $0,01 *$ & 0,98 & $0,02 *$ & $0,01 *$ \\
\hline ITMOVCOM & 0,00 & 24,42 & 0,00 & 14,80 & $0,00 *$ & $0,05 *$ & $0,00 *$ & $0,01 *$ \\
\hline ITRED & 0,00 & 18,39 & 0,00 & 28,95 & $0,00 *$ & $0,00 *$ & $0,00 *$ & 0,80 \\
\hline ITINT & 0,78 & 4,25 & $\mathbf{0 , 0 2 *}$ & --- & -- & 0,62 & $0,01 *$ & 0,14 \\
\hline ITTEMRED & 0,00 & 44,62 & 0,00 & $\mathrm{n} / \mathrm{d}$ & $\mathrm{n} / \mathrm{d}$ & $0,00 *$ & $0,00 *$ & 0,19 \\
\hline ITSEREQP & 0,00 & 7,28 & 0,00 & 10,64 & $0,00 *$ & 0,29 & $0,00 *$ & $0,00 *$ \\
\hline
\end{tabular}

De acordo com os resultados do teste ANOVA, os três grupos de empresas não apresentaram diferenças significativas quanto ao número de equipamentos por funcionário (ITEQPFUN), ficando a média geral da amostra em 0,30 equipamento por funcionário. Se forem considerados apenas os setores não produtivos (ITEQPADM), o teste aponta diferenças significativas entre as 
pequenas e as grandes e médias empresas. No caso destas últimas, esses valores ficaram acima de 1,0 equipamento por usuário; portanto nestes setores a diferença aponta a maior utilização de computadores do que no caso das pequenas empresas. De acordo com a variável ITMOV as empresas pequenas e médias têm percentagem menor de equipamentos móveis do que as grandes. Já de acordo com a variável ITMOVCOM, nas grandes empresas o número de equipamentos móveis por funcionário na área comercial aproxima-se do valor um, o que sugere que estes equipamentos provavelmente não são restritos a esta área.

A porcentagem de empresas médias e grandes que possuem redes (ITTEMRED) e a porcentagem de microcomputadores em rede nestas empresas (ITRED) são bastante semelhantes aos valores observados por Meirelles (2003). Entretanto, no caso das pequenas empresas, pôde-se verificar diferença estatisticamente significativa em relação a esses quesitos, mostrando que $38 \%$ destas empresas não possuem redes e as redes abrangem apenas $71 \%$ dos equipamentos disponíveis. No caso da quantidade de micros conectados à Internet (ITRED), as pequenas diferem estatisticamente das grandes e possuem maior percentual de equipamentos conectados. Isso possivelmente se deve ao uso nas grandes empresas de equipamentos em setores voltados exclusivamente ao processamento interno.

Nas pequenas empresas, a quantidade de computadores servidores por microcomputador (ITSEREQP) é o dobro do observado nas grandes. Uma possível explicação para essa diferença decorre das grandes poderem auferir ganhos de escala nessa utilização, por meio do uso de servidores mais potentes e pelo fato de as pequenas possuírem redes com menor número de micros, mas que exigem pelo menos um servidor. Quanto à forma de acesso à Internet, a Tabela 6 mostra que as grandes e médias empresas se conectam por meio de linhas de alta velocidade, enquanto quase a metade das pequenas ainda utiliza acesso por meio de linha telefônica discada com provedores pagos.

Tabela 6: Forma de Acesso à Internet

\begin{tabular}{|l|r|r|r|r|r|r|}
\hline \multirow{2}{*}{ Forma de Acesso } & \multicolumn{2}{|c|}{ Pequena } & \multicolumn{2}{c|}{ Média } & \multicolumn{2}{c|}{ Grande } \\
\cline { 2 - 7 } & Casos & \multicolumn{1}{c|}{$\%$} & Casos & $\%$ & Casos & \multicolumn{1}{c|}{$\%$} \\
\hline Provedor gratuito discado & 7 & 4,46 & 0 & 0,00 & 1 & 1,04 \\
\hline Provedor pago discado & 66 & 42,04 & 2 & 3,13 & 15 & 15,63 \\
\hline Linha até 256 kbps & 61 & 38,85 & 24 & 37,50 & 42 & 43,75 \\
\hline Linha até 512 kbps & 14 & 8,92 & 16 & 25,00 & 25 & 26,04 \\
\hline Linha acima de 512 kbps & 9 & 5,73 & 22 & 34,38 & 13 & 13,54 \\
\hline
\end{tabular}




\section{Dimensão Uso}

\section{Sistemas de Informações}

Na pesquisa solicitou-se às empresas que informassem a abrangência e a forma de obtenção dos sistemas de informação utilizados. Quanto à abrangência foi solicitado que as empresas informassem se possuíam ou não sistemas de informações ou módulos de sistemas integrados que abrangessem as áreas relacionadas no Quadro 1.

\section{Quadro 1: Tipos de Módulos e Sistemas de Informações}

\begin{tabular}{|l|l|}
\hline \multicolumn{1}{|c|}{ Integração Vertical e Horizontal } & \multicolumn{1}{c|}{ Integração Externa } \\
\hline - Faturamento e vendas & \\
- Contabilidade e livros fiscais & - Controle de importação e exportação \\
- Folha de pagamento e recursos humanos & - Comércio eletrônico via Internet \\
- Finanças (contas a pagar, a receber, tesouraria) & - Automação de força de vendas via computação móvel \\
- Controle da produção e estoques de produtos & - Relacionamento com clientes (CRM) \\
- Controle de compras e estoques de materiais & \\
\hline - Patrimônio (ativo fixo) & \multicolumn{1}{|c|}{ Apoio à Decisão } \\
\hline \multicolumn{1}{|c|}{ Automação de Processos } & - EIS (Executive Information Systems) \\
\hline - Controle de documentos e Workflow & - BI (Business Intelligence) \\
\hline
\end{tabular}

As empresas informaram o modo de obtenção de cada módulo de acordo com as seguintes categorias. (a) Desenvolvimento próprio: aplicativo desenvolvido por pessoal técnico pertencente ao quadro de funcionários da própria empresa. (b) Desenvolvimento terceirizado: aplicativo desenvolvido por pessoal especializado externo ou por empresa contratada. (c) Pacote isolado: aplicativo adquirido pronto de fornecedor especializado, contemplando apenas um tipo de função ou setor; por exemplo, pacote de contabilidade. (d) Pacote integrado (ERP): aplicativo adquirido pronto que atende, de maneira integrada, a mais de um tipo de função ou setor. Foram considerados como ERPs todos os tipos de pacotes informados como integrados, nacionais ou estrangeiros, independentemente do porte do fornecedor. Para esta análise o número de respostas válidas foi: 70 grandes, 92 médias e 134 pequenas.

A Tabela 7 apresenta as descrições e estatísticas das variáveis relativas a sistemas de informações e na tabela 8 constam os resultados dos testes das diferenças entre os grupos de empresas. De acordo com a variável SITRA, as empresas médias e grandes possuem mais módulos implementados do que as pequenas. Os números indicam 5,2 para as pequenas, 6,5 para as médias e 6,7 para as grandes, num total de sete possíveis. 


\section{Tabela 7: Variáveis de Sistemas de Informações}

\begin{tabular}{|c|c|c|c|c|c|c|c|c|c|c|}
\hline \multirow{2}{*}{ Variável } & \multirow{2}{*}{ Descrição } & \multicolumn{3}{|c|}{ Pequena } & \multicolumn{3}{|c|}{ Média } & \multicolumn{3}{|c|}{ Grande } \\
\hline & & $M$ & $S$ & $C A$ & $M$ & $S$ & $C A$ & $M$ & $S$ & $C A$ \\
\hline SITRA & $\begin{array}{c}\text { Número de sistemas transacionais } \\
\text { que a empresa possui }\end{array}$ & 5,2 & 1,9 & $-3,4$ & 6,5 & 1,1 & $-11,0$ & 6,7 & 0,8 & $-17,8$ \\
\hline SIFRO & $\begin{array}{c}\text { Número de sistemas front office } \\
\text { que a empresa possui }\end{array}$ & 0,6 & 1,0 & 10,3 & 1,0 & 1,1 & 5,1 & 1,4 & 1,3 & 1,8 \\
\hline SISIG & $\begin{array}{c}\% \text { de empresas que possuem } \\
\text { sistemas de informações gerenciais }\end{array}$ & $22 \%$ & & & $28 \%$ & & & $47 \%$ & & \\
\hline SIWKF & $\begin{array}{c}\% \text { de empresas que possuem } \\
\text { sistemas de workflow }\end{array}$ & $14 \%$ & & & $26 \%$ & & & $36 \%$ & & \\
\hline SITEMERP & $\begin{array}{l}\% \text { de empresas que possuem } \\
\text { ao menos um módulo ERP }\end{array}$ & $49 \%$ & & & $68 \%$ & & & $81 \%$ & & \\
\hline SIAPEERP & $\begin{array}{c}\% \text { de empresas que apenas } \\
\text { usam módulos ERP transacionais }\end{array}$ & $33 \%$ & & & $35 \%$ & & & $26 \%$ & & \\
\hline SITRAERP & $\begin{array}{c}\text { \% dos sistemas transacionais que são } \\
\text { módulos ERP na empresa }\end{array}$ & $41 \%$ & $47 \%$ & 1,7 & $57 \%$ & $43 \%$ & $-1,4$ & $64 \%$ & $39 \%$ & $-2,7$ \\
\hline SIFROERP & $\begin{array}{c}\text { \% dos sistemas de front office que são } \\
\text { módulos ERP na empresa }\end{array}$ & $13 \%$ & $34 \%$ & 10,4 & $21 \%$ & $40 \%$ & 5,8 & $20 \%$ & $37 \%$ & 5,2 \\
\hline
\end{tabular}

\section{Tabela 8: Testes de Significância das Variáveis de Sistemas de Informações}

\begin{tabular}{|c|c|c|c|c|c|c|c|c|}
\hline \multirow{2}{*}{ Variável } & \multirow{2}{*}{$\begin{array}{l}\text { Teste de } \\
\text { Levene }\end{array}$} & \multicolumn{2}{|c|}{ ANOVA } & \multicolumn{2}{|c|}{ Brown-Forsythe } & \multicolumn{3}{|c|}{ Games-Howell ou Tukey-Kramer } \\
\hline & & $F$ & Sig. & $F^{*}$ & Sig. & $P Q$ vs $M D$ & $P Q$ vs $G D$ & $M D$ vs $G D$ \\
\hline SITRA & 0,00 & 31,61 & 0,00 & 41,15 & $0,00 *$ & $0,00 *$ & $0,00^{*}$ & 0,17 \\
\hline SIFRO & 0,01 & 14,47 & 0,00 & 13,40 & $0,00 *$ & $0,02 *$ & $0,00 *$ & $0,05 *$ \\
\hline SISIG & 0,00 & 7,06 & 0,00 & 6,65 & $0,00^{*}$ & 0,59 & $0,00^{*}$ & $0,04 *$ \\
\hline SIWKF & 0,00 & 6,61 & 0,00 & 5,96 & $0,00^{*}$ & 0,08 & $0,00^{*}$ & 0,40 \\
\hline SITEMERP & 0,00 & 12,52 & 0,00 & 13,47 & $0,00 *$ & $0,01 *$ & $0,00^{*}$ & 0,14 \\
\hline SIAPEERP & 0,02 & 0,82 & 0,44 & 0,83 & 0,44 & --- & -- & -- \\
\hline SITRAERP & 0,00 & 7,15 & 0,00 & 7,59 & $0,00 *$ & $0,03^{*}$ & $0,00^{*}$ & 0,55 \\
\hline SIFROERP & 0,01 & 1,48 & 0,23 & 1,43 & 0,24 & --- & --- & -- \\
\hline
\end{tabular}

De acordo com a variável SITEMERP pode-se observar que $81 \%$ das grandes e 68\% das médias possuem, pelo menos, um módulo ERP instalado, resultado coerente com Meirelles (2003). Nas pequenas o índice de 49\% é superior ao obtido na pesquisa realizada em 2000 (FIESP/FIPE, 2000), em que apenas 19\% informaram possuir pacote comercial do tipo ERP. Isto pode indicar uma evolução no uso de pacotes pelas pequenas empresas nos últimos dois anos; entretanto o índice de utilização de sistemas ERP entre as pequenas ainda é significativamente menor do que nas médias e grandes empresas. A porcentagem de sistemas do tipo transacional, que são módulos ERP (SITRAERP), também é significativamente menor nas pequenas empresas.

A variável SIAPEERP mostra que a maioria das empresas não utiliza um ERP único e completo. Em geral elas combinam pacotes isolados e módulos desenvolvidos internamente ou por terceiros, independentemente do porte. No 
caso das grandes empresas isso, em parte, se justifica pelo uso de pacotes isolados de folha de pagamento e patrimônio, não disponíveis em alguns sistemas ERP estrangeiros. No caso dos módulos de front office, as empresas grandes destacaram-se diante das médias e pequenas (SIFRO); entretanto pode-se perceber que ainda é incipiente a implementação de módulos deste tipo (1,4 em 4 possíveis) e é reduzida a opção pela utilização de módulos de sistemas ERP para supri-la (SIFROERP).

A Figura 2 mostra o gráfico da porcentagem de módulos por forma de obtenção, considerando-se o total de módulos do tipo transacional informados pelas empresas. Nas grandes e médias empresas, há intensa participação de pacotes comerciais, ERP e isolados. Nas pequenas o quadro é distinto, havendo participação de apenas $40 \%$ de pacotes comerciais. Nas pequenas também se nota o maior índice de utilização de sistemas desenvolvidos por terceiros. Isso sugere uma característica das pequenas de procurarem implementar sistemas de informações que sejam espelhos da maneira como as coisas são feitas na empresa; por não disporem de recursos para manter analistas para o desenvolvimento interno, elas recorrem a terceiros para a realização desta tarefa.

\section{Figura 2: Forma de Obtenção dos Módulos Transacionais}

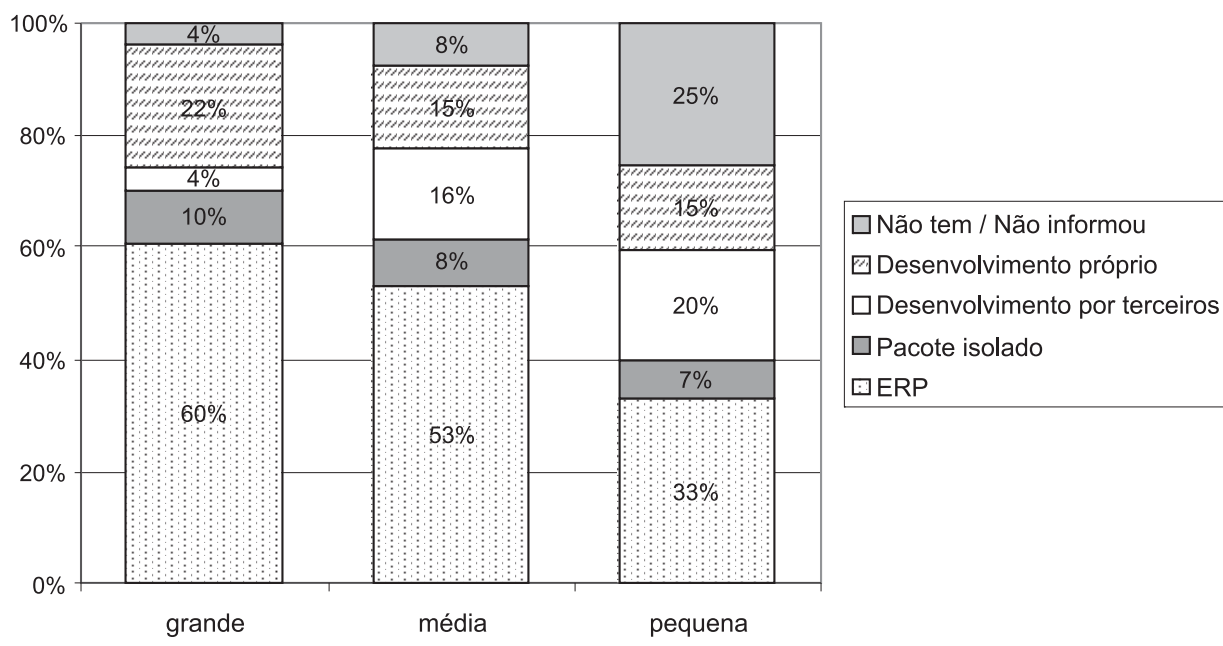

As empresas também foram solicitadas a informar sua percepção sobre a aderência e a dependência em relação aos sistemas que detinham, numa escala que varia de 1 (muito baixa) a 5 (muito alta). No caso da aderência, solicitou-se que ela fosse considerada alta, quando pouca ou nenhuma modificação foi necessária para o módulo se adaptar aos processos de negócio executados na empresa. No caso da dependência, solicitou-se que ela fosse considerada alta, quando no evento do sistema se tornar inoperante, as 
conseqüências afetariam drasticamente as operações da empresa. A Tabela 9 apresenta as variáveis pertinentes e os resultados dos testes de comparação entre os grupos, quando detectada diferença significativa pelos testes ANOVA ou Brown-Forsythe.

Tabela 9: Testes de Significância das Variáveis de Aderência e Dependência

\begin{tabular}{|c|c|c|c|c|c|c|c|c|c|c|c|}
\hline \multirow{2}{*}{ Variável } & \multirow{2}{*}{ Descrição } & \multicolumn{2}{|c|}{ Pequena } & \multicolumn{2}{|c|}{ Média } & \multicolumn{2}{|c|}{ Grande } & \multirow{2}{*}{$\begin{array}{l}\text { Sig. de } \\
\text { Fou } F^{*}\end{array}$} & \multirow{2}{*}{$\begin{array}{l}P Q v s \\
M D\end{array}$} & \multirow{2}{*}{$\stackrel{P Q v s}{G D}$} & \multirow{2}{*}{$\begin{array}{c}M D v s \\
G D\end{array}$} \\
\hline & & $M$ & $n$ & $M$ & $n$ & $M$ & $n$ & & & & \\
\hline SITRADEP & $\begin{array}{c}\text { Dependência dos } \\
\text { módulos transacionais }\end{array}$ & 3,50 & 111 & 3,71 & 86 & 4,09 & 62 & $0,00 *$ & 0,33 & $0,00^{*}$ & $0,02 *$ \\
\hline SITRAADE & $\begin{array}{c}\text { Aderência dos } \\
\text { módulos transacionais }\end{array}$ & 3,54 & 117 & 3,70 & 84 & 3,94 & 62 & $0,01 *$ & 0,44 & $0,00 *$ & 0,14 \\
\hline SIFRODEP & $\begin{array}{l}\text { Dependência dos } \\
\text { módulos de front office }\end{array}$ & 2,80 & 46 & 3,08 & 46 & 3,52 & 44 & 0,15 & --- & --- & --- \\
\hline SIFROADE & $\begin{array}{l}\text { Aderência dos } \\
\text { módulos de front office }\end{array}$ & 3,05 & 47 & 3,43 & 46 & 3,42 & 46 & 0,24 & -- & --- & --- \\
\hline
\end{tabular}

A dependência das operações das empresas dos sistemas transacionais (SITRADEP) está de acordo com o esperado, pois ela é crescente em relação ao porte das empresas; notar que estatisticamente as pequenas e médias se diferenciam das grandes empresas. Isso indica que os sistemas de informações apóiam os processos empresariais de maneira efetiva nas grandes empresas. A aderência desses sistemas (SITRAADE) também se mostrou crescente com o porte, mas em menor grau; a diferença entre pequenas e médias e médias e grandes não se mostrou estatisticamente significativa. Para os módulos de front office, tanto a sua dependência (SIFRODEP) quanto a sua aderência (SIFROADE) não mostraram diferenças estatisticamente significativas entre os três grupos. Observar que apenas um reduzido número de empresas informou possuir estes módulos.

\section{Negócios Eletrônicos}

$\mathrm{Na}$ pesquisa as empresas forneceram dados sobre o uso da Internet para os seus negócios. As variáveis envolvidas estão apresentadas na Tabela 10; na Tabela 11 constam os resultados dos testes das diferenças entre os grupos de empresas. 


\section{Tabela 10: Variáveis de Negócios Eletrônicos}

\begin{tabular}{|c|c|c|c|c|c|c|c|c|c|c|c|c|c|}
\hline \multirow{2}{*}{ Variável } & \multirow{2}{*}{ Descrição } & \multicolumn{4}{|c|}{ Pequena } & \multicolumn{4}{|c|}{ Média } & \multicolumn{4}{|c|}{ Grande } \\
\hline & & $M$ & $S$ & $C A$ & $n$ & $M$ & $S$ & $C A$ & $n$ & $M$ & $S$ & $C A$ & $n$ \\
\hline $\mathrm{B} 2 \mathrm{~B} \%$ & $\begin{array}{l}\% \text { do faturamento } \\
\text { realizado via B2B }\end{array}$ & 12,5 & 17,9 & 7,9 & 68 & 15,9 & 25,3 & 5,6 & 45 & 19,7 & 30,0 & 4,6 & 44 \\
\hline $\mathrm{B} 2 \mathrm{~S} \%$ & $\begin{array}{c}\% \text { das compras } \\
\text { realizadas via } \mathrm{B} 2 \mathrm{~B}\end{array}$ & 13,3 & 19,2 & 9,2 & 69 & 7,9 & 12,5 & 6,7 & 46 & 15,6 & 23,0 & 4,7 & 45 \\
\hline $\mathrm{B} 2 \mathrm{C} \%$ & $\begin{array}{l}\% \text { do faturamento } \\
\text { realizado via B2C }\end{array}$ & 7,2 & 10,7 & 8,9 & 68 & 3,2 & 7,0 & 6,7 & 46 & 2,5 & 12,4 & 16,8 & 44 \\
\hline EMAIL & $\begin{array}{l}\text { Uso do e-mail } \\
\text { para negócios }\end{array}$ & 5,5 & 1,9 & 1,4 & 148 & 6,3 & 1,8 & $-0,5$ & 93 & 6,9 & 2,0 & $-2,1$ & 69 \\
\hline WEB & $\begin{array}{l}\text { Uso do web site da } \\
\text { empresa para negócios }\end{array}$ & 2,3 & 1,7 & 3,8 & 145 & 2,6 & 1,6 & 3,1 & 94 & 3,4 & 2,2 & 2,3 & 68 \\
\hline
\end{tabular}

Segundo a Tabela 11, o faturamento e as compras via B2B (B2B\% e B2S\%) não apresentaram diferenças estatisticamente significativas entre os grupos. Embora os valores sejam diferentes, principalmente no caso de compras das empresas médias, essas variáveis apresentaram elevada dispersão e casos extremos em cada um dos grupos, mas que, em primeira análise, não puderam ser excluídos da amostra. Para a variável B2B\% a média geral verificada entre as 157 empresas que responderam aos quesitos de negócios eletrônicos foi de 15,5\%. Para a variável B2S\% a média observada foi de $12,4 \%$. No caso da venda direta ao consumidor (B2C\%), pode-se detectar apenas a diferença entre as pequenas e as grandes, tendo as primeiras maiores porcentagens de seu faturamento realizadas por esse meio (7,3\% contra 2,5\%). Este é um resultado interessante e eventualmente decorre do fato de as grandes empresas, em geral, não realizarem vendas diretas ao consumidor final.

\section{Tabela 11: Testes de Significância das Variáveis de Negócios Eletrônicos}

\begin{tabular}{|c|c|c|c|c|c|c|c|c|}
\hline \multirow{2}{*}{ Variável } & \multirow{2}{*}{$\begin{array}{c}\text { Teste de } \\
\text { Levene }\end{array}$} & \multicolumn{2}{|c|}{ ANOVA } & \multicolumn{2}{|c|}{ Brown-Forsythe } & \multicolumn{3}{|c|}{ Games-Howell ou Tukey-Kramer } \\
\hline & & $\mathrm{F}$ & Sig. & $\mathrm{F}^{*}$ & Sig. & $P Q$ vs $M D$ & $P Q$ vs $G D$ & $M D$ vs $G D$ \\
\hline $\mathrm{B} 2 \mathrm{~B} \%$ & 0,01 & 1,21 & 0,30 & 1,08 & 0,34 & --- & --- & --- \\
\hline $\mathrm{B} 2 \mathrm{~S} \%$ & 0,00 & 2,05 & 0,13 & 2,07 & 0,13 & --- & --- & --- \\
\hline $\mathrm{B} 2 \mathrm{C} \%$ & 0,12 & 3,54 & $\mathbf{0 , 0 3}$ & --- & --- & 0,10 & 0,05 & 0,95 \\
\hline EMAIL & 0,99 & 14,65 & $\mathbf{0 , 0 0}$ & --- & --- & $\mathbf{0 , 0 0}$ & $\mathbf{0 , 0 0}$ & 0,10 \\
\hline WEB & 0,01 & 7,31 & 0,00 & 6,77 & $\mathbf{0 , 0 0}$ & 0,56 & $\mathbf{0 , 0 0}$ & 0,03 \\
\hline
\end{tabular}

A variável EMAIL representa a soma da importância atribuída pelos respondentes a dois quesitos: uso do e-mail para realização de negócios e para os funcionários realizarem suas atividades. As empresas médias e grandes atribuíram maior importância ao uso do correio eletrônico do que as pequenas. A variável WEB indica quantas funções em um grupo de 8, indicadas na Tabela 12, estão disponíveis no web site da empresa. A utilização é pequena, mesmo nas 
empresas grandes, com média de 3,4 funções em 8 possíveis. A Tabela 12 apresenta o detalhamento completo da variável WEB. A maioria das empresas, independentemente do porte, ainda restringe o uso de seu web site à apresentação de informações institucionais e de informações sobre seus produtos e serviços. Cerca de 35\% das empresas já usa o web site para o atendimento a clientes e é de se supor que este percentual tende a aumentar.

Tabela 12: Funções do Web site das Empresas

\begin{tabular}{|c|c|c|c|c|c|c|c|c|c|c|}
\hline Tamanho & Dados & 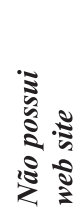 & 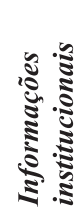 & 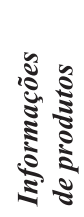 & 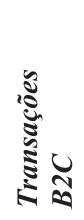 & 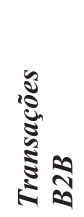 & 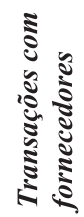 & 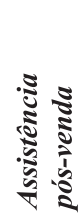 & 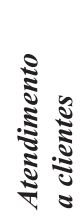 & 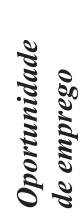 \\
\hline \multirow[t]{2}{*}{ Grande } & $\%$ & 11,29 & 85,48 & 80,65 & 16,13 & 33,87 & 29,03 & 22,58 & 25,81 & 32,26 \\
\hline & Casos & 62 & 62 & 62 & 62 & 62 & 62 & 62 & 62 & 62 \\
\hline \multirow[t]{2}{*}{ Média } & $\%$ & 10,42 & 86,46 & 84,38 & 7,29 & 16,67 & 5,21 & 12,50 & 39,58 & 9,38 \\
\hline & Casos & 96 & 96 & 96 & 96 & 96 & 96 & 96 & 96 & 96 \\
\hline \multirow[t]{2}{*}{ Pequena } & $\%$ & 19,61 & 63,40 & 77,78 & 13,73 & 17,65 & 11,11 & 13,07 & 34,64 & 4,58 \\
\hline & Casos & 153 & 153 & 153 & 153 & 153 & 153 & 153 & 153 & 153 \\
\hline
\end{tabular}

\section{Dimensão Gestão}

\section{Recursos Humanos da Área de TI}

As empresas informaram a quantidade de funcionários e terceiros em tempo integral na área de TI, observando que, por exemplo, duas pessoas em meio período foram consideradas como uma em tempo integral. Nas Tabelas 13 e 14 constam as variáveis de interesse e na Tabela 15 constam os testes estatísticos para as diferenças entre os grupos.

\section{Tabela 13: Variáveis de Recursos Humanos de TI}

\begin{tabular}{|c|c|c|c|c|c|c|c|c|c|c|c|c|c|c|c|c|c|c|c|}
\hline \multirow{2}{*}{ Variável } & \multirow{2}{*}{ Descrição } & \multicolumn{6}{|c|}{ Pequena } & \multicolumn{6}{|c|}{ Média } & \multicolumn{6}{|c|}{ Grande } \\
\hline & & $M$ & $S$ & $C A$ & min & máx & $n$ & $M$ & $S$ & $C A$ & $\min$ & máx & $n$ & $M$ & $S$ & $C A$ & $\min$ & máx & $n$ \\
\hline PETOT & $\begin{array}{c}\text { Pessoal de TI } \\
\text { total }\end{array}$ & 1,7 & 1,8 & 7,0 & 0 & 9 & 124 & 4,1 & 3,2 & 8,4 & 0 & 19 & 87 & 22,7 & 27,6 & 8,2 & 2 & 142 & 67 \\
\hline PEFUN & \begin{tabular}{|c|} 
Pessoal de TI \\
funcionários
\end{tabular} & 1,0 & 1,3 & 10,5 & 0 & 8 & 124 & 3,0 & 2,7 & 7,4 & 0 & 17 & 87 & 18,1 & 22,3 & 6,7 & 0 & 98 & 67 \\
\hline PETER & $\begin{array}{c}\text { Pessoal de TI } \\
\text { terceirizado }\end{array}$ & 0,7 & 1,1 & 7,3 & 0 & 5 & 124 & 1,1 & 1,6 & 7,6 & 0 & 8 & 87 & 4,5 & 10,5 & 14 , & 0 & 67 & 67 \\
\hline
\end{tabular}


Tabela 14: Variáveis de Recursos Humanos de TI

\begin{tabular}{|c|c|c|c|c|c|c|c|c|c|c|c|c|c|}
\hline \multirow{2}{*}{ Variável } & Descrição & \multicolumn{3}{|c|}{ Pequena } & \multicolumn{5}{c|}{ Média } & \multicolumn{4}{c|}{ Grande } \\
\cline { 3 - 15 } & $M$ & $S$ & $C A$ & $n$ & $M$ & $S$ & $C A$ & $n$ & $M$ & $S$ & $C A$ & $n$ \\
\hline PETERPER & $\begin{array}{c}\text { Pessoal de TI terceirizado } \\
\%\end{array}$ & $24 \%$ & $36 \%$ & 5,3 & 124 & $26 \%$ & $33 \%$ & 3,7 & 87 & $20 \%$ & $26 \%$ & 3,9 & 67 \\
\hline PEATI & $\begin{array}{c}\text { Atividades da } \\
\text { equipe interna de TI }\end{array}$ & 1,13 & 1,00 & 2,2 & 149 & 1,71 & 1,0 & $-0,9$ & 99 & 2,08 & 1,04 & $-3,2$ & 78 \\
\hline PEPLA & $\begin{array}{c}\text { TI compartilha planejamento } \\
\text { com outras áreas }\end{array}$ & $31 \%$ & & & 149 & $39 \%$ & & & 99 & $46 \%$ & & & 78 \\
\hline PEPORTOT & $\begin{array}{c}\text { Pessoal de TI por } \\
\text { funcionário da empresa }\end{array}$ & 0,04 & 0,05 & 7,8 & 120 & 0,02 & 0,0 & 6,2 & 82 & 0,01 & 0,01 & 4,3 & 61 \\
\hline PEPORADM & $\begin{array}{c}\text { Pessoal de TI por funcionário } \\
\text { exceto produção }\end{array}$ & 0,12 & 0,14 & 6,9 & 118 & 0,10 & 0,1 & 8,0 & 79 & 0,07 & 0,05 & 4,9 & 53 \\
\hline PEPORMIC & $\begin{array}{c}\text { Pessoal de TI } \\
\text { por micro }\end{array}$ & 0,15 & 0,22 & 14,4 & 120 & 0,08 & 0,1 & 7,1 & 86 & 0,05 & 0,04 & 0,0 & 67 \\
\hline
\end{tabular}

\section{Tabela 15: Testes de Significância das Variáveis de Recursos Humanos de TI}

\begin{tabular}{|c|c|c|c|c|c|c|c|c|}
\hline \multirow{2}{*}{ Variável } & \multirow{2}{*}{$\begin{array}{l}\text { Teste de } \\
\text { Levene }\end{array}$} & \multicolumn{2}{|c|}{$A N O V A$} & \multicolumn{2}{|c|}{ Brown-Forsythe } & \multicolumn{3}{|c|}{ Games-Howell ou Tukey-Kramer } \\
\hline & & $F$ & Sig. & $F^{*}$ & Sig. & $P Q$ vs $M D$ & $P Q$ vs $G D$ & $M D$ vs $G D$ \\
\hline PETERPER & 0,00 & 0,65 & 0,52 & 0,71 & 0,49 & --- & --- & -- \\
\hline PEATI & 0,53 & 24,12 & $0,00 *$ & --- & --- & $\mathbf{0 , 0 0 *}$ & $\mathbf{0 , 0 0 *}$ & $0,05 *$ \\
\hline PEPLA & 0,00 & 2,74 & 0,07 & 2,67 & 0,07 & --- & --- & --- \\
\hline PEPORTOT & 0,00 & 16,03 & 0,00 & 25,15 & $0,00 *$ & $\mathbf{0 , 0 0 *}$ & $\mathbf{0 , 0 0 *}$ & $0,00 *$ \\
\hline PEPORADM & 0,00 & 4,77 & 0,01 & 6,69 & $\mathbf{0 , 0 0 *}$ & 0,43 & $0,00 *$ & $0,01 *$ \\
\hline PEPORMIC & 0,00 & 10,31 & 0,00 & 15,09 & $0,00 *$ & $0,01 *$ & $0,00 *$ & $0,00 *$ \\
\hline
\end{tabular}

Os resultados apontam para departamentos de TI bastante enxutos; entretanto as medidas acima possuem grau de dispersão considerável nos três portes de empresa. Por exemplo, na distribuição das empresas grandes, a média é de 22,7 pessoas, mas o desvio padrão é de 27,6 pessoas e o valor máximo informado é de 142 pessoas. Por outro lado, a assimetria é positiva nos três portes, o que indica uma concentração nos valores menores da distribuição. Nas pequenas também se percebe a distribuição assimétrica ao lado esquerdo indicando que elas possuem um ou nenhum profissional dedicado em tempo integral à área de TI.

Observa-se semelhança na proporção de terceiros na equipe de TI entre os três portes de empresas (PETERPER) com média geral de 24\%. Isso sugere o incremento da utilização de terceiros pelas grandes empresas, que tradicionalmente operavam com equipes internas. A variável PEATI mede o número de tipos de atividades realizadas pela equipe interna de TI (funcionários e terceiros), considerando o desenvolvimento de aplicativos tradicionais, o desenvolvimento de aplicativos para Internet e as atividades de suporte. Nesse caso observou-se que com o aumento do porte da empresa mais atividades são realizadas internamente. 
A partir da variável PEPLA, observa-se pouco envolvimento de outras áreas no planejamento de TI. Embora o resultado do teste tenha mostrado semelhança entre os portes, no nível de significância de 7\%, os resultados sugerem aumento da participação das áreas com o aumento do porte; entretanto, mesmo no caso das grandes, menos da metade das empresas informou envolver outras áreas no planejamento de TI, um resultado contrário ao inicialmente esperado para esse porte de empresas.

A partir das variáveis PEPORTOT, PEPORADM e PEPORMIC, observa-se uma redução do número de funcionários de TI por funcionário da empresa (total ou excluindo a produção) e por quantidade de micros, com o aumento do porte da empresa. Novamente essa relação pode estar associada ao ganho de escala com os recursos humanos de TI em empresas maiores. Em princípio isso não significa que os usuários de empresas pequenas e médias sejam mais bem atendidos, mas que ocorre o uso mais produtivo dos recursos humanos de TI nas empresas maiores.

\section{Investimentos da Área de TI}

Nas Tabelas 16 e 17 constam as variáveis relacionadas ao investimento e despesas da área de TI. Foram considerados apenas os investimentos informados para o ano 2001, pois a informação fornecida pelas empresas para o ano de 2002 se tratava de uma projeção.

Tabela 16: Variáveis de Investimentos de TI

\begin{tabular}{|c|c|c|c|c|c|c|c|c|c|c|c|c|c|}
\hline \multirow{2}{*}{ Variável } & \multirow{2}{*}{ Descrição } & \multicolumn{4}{|c|}{ Pequena } & \multicolumn{4}{|c|}{ Média } & \multicolumn{4}{|c|}{ Grande } \\
\hline & & $M$ & $S$ & $C A$ & $n$ & $M$ & $S$ & $C A$ & $n$ & $M$ & $S$ & $C A$ & $n$ \\
\hline ININV & $\begin{array}{l}\text { Investimentos em TI } \\
\text { (em } R \$ \text { mil) }\end{array}$ & 21,9 & 25,3 & 5,5 & 77 & 144,6 & 241,7 & 12,1 & 60 & 1513,4 & 3623,2 & 13,2 & 36 \\
\hline INDES & $\begin{array}{c}\text { Despesas de TI } \\
(\text { em } R \$ \mathrm{mil})\end{array}$ & 11,8 & 13,7 & 5,6 & 59 & 100,0 & 317,0 & 22,2 & 60 & 1826,1 & 3858,5 & 10,7 & 35 \\
\hline ININVDES & $\begin{array}{l}\text { Investimento e despesas } \\
\text { como } \% \text { do faturamento }\end{array}$ & $1,3 \%$ & $1,5 \%$ & 8,7 & 53 & $1,0 \%$ & $2,3 \%$ & 17,0 & 49 & $1,1 \%$ & $1,7 \%$ & 11,3 & 32 \\
\hline INDESMIC & $\begin{array}{c}\text { Despesas de TI por micro } \\
\text { (em } R \$ \text { mil) }\end{array}$ & 0,9 & 0,8 & 3,8 & 56 & 1,2 & 1,5 & 10,5 & 59 & 2,7 & 2,1 & 1,2 & 35 \\
\hline
\end{tabular}

\section{Tabela 17: Testes de Significância das} Variáveis de Investimentos de TI

\begin{tabular}{|c|c|c|c|c|c|c|c|c|}
\hline \multirow{2}{*}{ Variável } & \multirow{2}{*}{$\begin{array}{l}\text { Teste de } \\
\text { Levene }\end{array}$} & \multicolumn{2}{|c|}{ ANOVA } & \multicolumn{2}{|c|}{ Brown-Forsythe } & \multicolumn{3}{|c|}{ Games-Howell ou Tukey-Kramer } \\
\hline & & $F$ & Sig. & $F^{*}$ & Sig. & $P Q v s M D$ & $P Q v_{s} G D$ & $M D$ vs $G D$ \\
\hline ININVDES & 0,05 & 0,359 & 0,699 & 0,368 & 0,693 & --- & -- & -- \\
\hline INDESMIC & 0,00 & 17,070 & 0,000 & 14,320 & $0,00 *$ & 0,60 & $0,00 *$ & $0,00 *$ \\
\hline
\end{tabular}


Os investimentos e despesas de TI na forma de porcentagem do faturamento (ININVDES) não apresentaram diferenças estatisticamente significativas, ficando a média geral das 134 empresas que forneceram essa informação em torno de 1,1\%. Por outro lado, a despesa de TI dividida pela quantidade de microcomputadores (INDESMIC) é significativamente maior nas empresas grandes. Apesar da constatação anterior de que a quantidade de pessoal de TI por microcomputador diminui com o porte, o custo total por equipamento aumenta com o porte.

\section{Impactos do Uso de Tecnologia da Informação}

As empresas também avaliaram os impactos do uso da TI, indicando o seu grau de concordância com as frases sintetizadas na descrição das variáveis da Tabela 18, usando a escala de 1 (discordo totalmente) até 5 (concordo totalmente). Notar que os valores da tabela inferiores a 3 indicam pouco impacto, enquanto os valores superiores indicam maior impacto. Na Tabela 19 constam os testes estatísticos para as diferenças entre os grupos de empresas.

Tabela 18: Variáveis de Impacto da TI

\begin{tabular}{|c|c|c|c|c|c|c|c|c|c|c|c|c|c|}
\hline \multirow{2}{*}{ Variável } & Descrição & \multicolumn{3}{|c|}{ Pequena } & \multicolumn{1}{c|}{ Média } & \multicolumn{5}{c|}{ Grande } \\
\cline { 3 - 16 } IMVEN & $\begin{array}{c}\text { A TI contribuiu para o } \\
\text { aumento das vendas }\end{array}$ & 3,1 & 1,3 & $-0,2$ & 149 & 3,2 & 1,2 & $-1,3$ & 99 & 3,5 & 1,1 & $-2,3$ & 78 \\
\hline IMPES & $\begin{array}{c}\text { A TI contribuiu para a } \\
\text { redução de pessoal }\end{array}$ & 2,8 & 1,3 & 0,5 & 149 & 2,8 & 1,2 & 0,0 & 99 & 3,5 & 1,0 & $-1,1$ & 78 \\
\hline IMEST & $\begin{array}{c}\text { A TI contribuiu para a } \\
\text { redução de estoques }\end{array}$ & 2,7 & 1,3 & 1,2 & 149 & 3,1 & 1,3 & $-1,1$ & 99 & 3,6 & 1,0 & $-2,1$ & 78 \\
\hline IMPRO & $\begin{array}{c}\text { A TI contribuiu para a } \\
\text { melhoria dos produtos e serviços }\end{array}$ & 3,5 & 1,2 & $-2,4$ & 149 & 3,5 & 1,1 & $-2,4$ & 99 & 3,6 & 1,0 & $-2,7$ & 78 \\
\hline IMCLI & $\begin{array}{c}\text { A TI contribuiu para a } \\
\text { melhoria do atendimento ao cliente }\end{array}$ & 3,8 & 1,1 & $-4,0$ & 149 & 3,9 & 0,9 & $-3,2$ & 99 & 4,0 & 0,9 & $-3,5$ & 78 \\
\hline IMDEC & $\begin{array}{c}\text { A TI contribuiu para a } \\
\text { melhoria da tomada de decisão }\end{array}$ & 3,7 & 1,1 & $-3,7$ & 149 & 3,8 & 1,0 & $-3,9$ & 99 & 4,1 & 0,8 & $-2,6$ & 78 \\
\hline IMOPE & $\begin{array}{c}\text { A TI contribuiu para a } \\
\text { redução de problemas operacionais }\end{array}$ & 3,3 & 1,1 & $-1,9$ & 149 & 3,6 & 0,9 & $-2,0$ & 99 & 3,9 & 0,9 & $-3,4$ & 78 \\
\hline IMLUC & $\begin{array}{c}\text { A TI contribuiu para o } \\
\text { aumento dos lucros }\end{array}$ & 2,9 & 1,3 & 0,4 & 149 & 3,1 & 1,1 & $-1,4$ & 99 & 3,4 & 0,9 & $-1,9$ & 78 \\
\hline
\end{tabular}

Tabela 19: Testes de Significância das Variáveis de Impacto da TI

\begin{tabular}{|c|c|c|c|c|c|c|c|c|}
\hline \multirow{2}{*}{ Variável } & \multirow{2}{*}{$\begin{array}{l}\text { Teste de } \\
\text { Levene }\end{array}$} & \multicolumn{2}{|c|}{ ANOVA } & \multicolumn{2}{|c|}{ Brown-Forsythe } & \multicolumn{3}{|c|}{ Games-Howell or Tukey-Kramer } \\
\hline & & $F$ & Sig. & $F^{*}$ & Sig. & $P Q$ vs $M D$ & $P Q$ vs $G D$ & $M D$ vs $G D$ \\
\hline IMVEN & 0,44 & 3,12 & 0,05 & & & 0,98 & 0,05 & 0,10 \\
\hline IMPES & 0,01 & 9,00 & 0,00 & 9,70 & $\mathbf{0 , 0 0}$ & 0,97 & $\mathbf{0 , 0 0}$ & $\mathbf{0 , 0 0}$ \\
\hline IMEST & 0,01 & 15,77 & 0,00 & 16,92 & $\mathbf{0 , 0 0}$ & $\mathbf{0 , 0 4}$ & $\mathbf{0 , 0 0}$ & $\mathbf{0 , 0 0}$ \\
\hline IMPRO & 0,13 & 0,13 & 0,88 & & & & & \\
\hline IMCLI & 0,00 & 0,87 & 0,42 & 0,95 & 0,39 & & & \\
\hline IMDEC & 0,01 & 3,32 & 0,04 & 3,62 & $\mathbf{0 , 0 3}$ & 0,81 & $\mathbf{0 , 0 2}$ & 0,11 \\
\hline IMOPE & 0,00 & 10,90 & 0,00 & 11,99 & $\mathbf{0 , 0 0}$ & 0,12 & $\mathbf{0 , 0 0}$ & $\mathbf{0 , 0 1}$ \\
\hline IMLUC & 0,01 & 5,03 & 0,01 & 5,57 & $\mathbf{0 , 0 0}$ & 0,18 & $\mathbf{0 , 0 0}$ & 0,29 \\
\hline \multicolumn{9}{|c|}{ * diferença estatisticamente significativa ao nível de $5 \%$} \\
\hline
\end{tabular}


O impacto é considerado relativamente maior pelas grandes empresas em todos os itens pesquisados, particularmente na qualidade da tomada de decisão e atendimento ao cliente. Os resultados sugerem que as empresas menores não conseguem reduções de pessoal, o que vai de encontro às expectativas mais imediatas destas empresas. Pode-se argumentar se a informatização para este porte de empresas necessariamente deveria implicar impactos significativos de curto prazo. O fato de o impacto do uso de TI ser considerado menor, especialmente nos itens redução de pessoal, redução de estoques e aumento dos lucros, pode indicar que quanto maior a empresa, mais complexos são seus negócios e, conseqüentemente, maior o impacto do uso de TI.

\section{Conclusões}

A avaliação da dimensão infra-estrutura de tecnologia forneceu poucos subsídios para a diferenciação dos níveis de informatização das empresas. De modo geral, qualquer que seja seu porte, as empresas parecem estar satisfatoriamente supridas por equipamentos. Como era de se esperar, as empresas menores possuem menor suporte de redes de comunicações e significativamente menos equipamentos móveis; entretanto isso não significa necessariamente uma deficiência, pois para este porte de empresa possivelmente os procedimentos comerciais não são beneficiados por meio do uso desses recursos.

Com relação à dimensão uso, há diferenças significativas segundo o porte das empresas no número de SI's que elas utilizam; entretanto essa diferença se atenua muito no caso de sistemas transacionais, sugerindo que, para as necessidades mais evidentes, as empresas estão bem supridas. A integração destes sistemas é menor nas empresas menores, o que, em princípio, pode não ser satisfatório e estar associado aos custos deste tipo de abordagem para SI's. Sistemas de workflow e de informações gerencias também são pouco utilizados por estas empresas. A dependência das empresas de seus SI's é muito maior nas grandes empresas, o que sugere que as empresas menores eventualmente podem prescindir dos SI’s que atualmente detêm. Em relação à aderência dos sistemas à empresa, não se observa diferença significativa entre os portes; mas as respostas sugerem que essa aderência em geral deixa a desejar. No âmbito de negócios eletrônicos, os resultados são pouco expressivos, mas a grande variabilidade nas respostas sugere que as possibilidades neste contexto ainda estão sendo exploradas pelas empresas. $\mathrm{O}$ uso intensivo do correio eletrônico nos negócios atesta as possibilidades do uso intensivo de sistemas baseados na web, particularmente pelas empresas menores. 
Com relação à dimensão gestão, as áreas de TI das empresas podem ser consideradas reduzidas. Cerca de um quarto do pessoal de TI é terceirizado e as empresas maiores mantêm equipes significativamente maiores. Também se pode supor que o perfil dos profissionais que atuam em pequenas, médias e grandes empresas é muito diferente, tendendo para melhor qualificação nas grandes. Nas empresas pequenas, a área de TI é bem caracterizada e tende a ser constituída por uma única pessoa. Nestas empresas a terceirização da TI é maior do que nas grandes empresas, o que provavelmente decorre do custo para manter equipes internas e do barateamento do preço de pacotes e da sua manutenção por terceiros. Os investimentos mais despesas de TI são homogêneos entre os portes das empresas; mas as empresas maiores possuem nível de despesas de TI proporcionalmente maior. Claramente as despesas compensam para estas empresas.

Os resultados da pesquisa sugerem que, conforme aumenta o tamanho da empresa, tanto maior é a sua dependência da TI e tanto melhor a tecnologia que acaba sendo utilizada. Também mostram que as empresas maiores auferem ganhos de escala muito mais evidentes que as menores. No caso das empresas menores o custo do uso da TI aparenta ser alto para elas e, em sentido amplo, o ganho de qualidade é proporcionalmente menor. Parte desses efeitos pode ser atribuída à falta de integração dos processos e sistemas nas pequenas empresas. Com base nessa interpretação deve existir um nível de porte da empresa, a partir do qual ela passa a obter ganhos compensatórios baseados no uso da TI. O dilema é que o crescimento da empresa até este ponto acaba não sendo favorecido pelo uso da TI.

Há tentativas para minorar este dilema; por exemplo, a solução ASP (Application Sevice Provider) tem sido considerada uma alternativa efetiva para as pequenas empresas. O fornecedor de TI fornece serviços remotos de sistemas de informações, sem que a empresa precise manter uma infra-estrutura própria de tecnologia e pessoal; entretanto essa solução exige organização e aderência a processos bem definidos de trabalho. Dessa forma, a flexibilidade, que pode constituir a única vantagem competitiva de muitas pequenas empresas, acaba cerceada e a empresa novamente não irá auferir ganhos com o uso da TI.

Os resultados da pesquisa mostram que a informatização é perseguida pelas empresas de todos os portes. Em princípio, não é possível estabelecer que essa informatização tenha atingido nas pequenas e médias empresas níveis que correspondam, conforme definição proposta, ao uso gerenciado de recursos de TI para apoiar e desenvolver uma organização, visando otimizar o seu desempenho. Em síntese se observa que quanto mais intenso é o uso da TI, tanto maior é considerado o seu impacto e melhor é considerado o desempenho das 
empresas. Isto corrobora Premkumar (2003) que estabelece que a utilidade da tecnologia aumenta na medida em que mais pessoas e empresas a adotam. Isso também sugere que externalidades de rede condicionam o incremento do uso da TI e de seu sucesso. Por enquanto, estas observações dizem respeito principalmente às empresas de maior porte.

Pode ser considerado como adequado o modelo de dimensões da informatização, proposto originalmente, visto que em todas as dimensões, com maior ou menor grau de segurança, foi possível detectar diferenças significativas entre as variáveis pesquisadas; mas também é necessário precaução, pois em alguns casos as dispersões observadas podem sugerir que as variáveis pesquisadas possivelmente não são entendidas e interpretadas da mesma forma pelas empresas, e em particular pelos respondentes. Isso exige detalhamento maior em determinadas perguntas e correspondentes análises. Também existem várias questões de difícil tratamento e avaliação; por exemplo, os diferentes graus de sofisticação da TI nas empresas e que afetam de forma distinta os diversos estratos do modelo. A discussão destas e outras questões não foi objetivo deste trabalho, mas certamente deve comparecer num eventual modelo de avaliação da informatização de empresas.

Artigo recebido em 02.10.2003. Aprovado em 12.03.2004.

\section{ReferênCias Bibliográficas}

AZAD, A. N.;

ERDEM,A.S.;

SALEEM, N.

A framework for realizing the potential of information technology in developing countries. International Journal of Commerce and Management, Indiana, n.8, v.2, p. 121133, 1998.

\section{DOLL, W. J.;}

TORKZADEH, G.

Developing a multidimensional measure of system use in an organizational context. Information \& Management, Amsterdã: Elsevier Sequoia, v. 33, n. 1, p.171-185. 1998.
FIESP/FIPE.

Perfil da Indústria Digital. 2000. Pesquisas_fiesp. Disponível em: $<$ http://www.fiesp.com.br/>. Acesso em: 27 abr. 2003.

GARSON, D.

PA765 Statnotes: An Online Textbook. 2003. Disponível em: <http:// www2.chass.ncsu.edu/ garson/pa765/anova.htm>. Acesso em: 17 apr. 2003.

GLASS, G. V.; HOPKINS, K. D.

Statistical Methods in Education and Psychology. 3. ed. Boston:Allyn and Bacon. p. 1996, 674. 
LAUDON, K. C.;

LAUDON, J.P.

Management Information Systems.

7. ed. Upper Saddle River: Prentice Hall. 2001, p. 547.

LIM, S. K.

A framework to evaluate the informatization level.. In: GREMBERG, W. (Ed.). Information Technology Evaluation: methods \& management. 1. ed. Hershey: IGP. p. 2001, 279.

LUCAS, H. C.

Information Technology for Management, 6. ed. New York: McGraw-Hill. 1997. 714p.

\section{MEIRELLES, F.}

Pesquisa Administração de Recursos de Informática, 14. ed. São Paulo: Fundação Getúlio Vargas. 2003. p. 87.
PREMKUMAR. G.

A meta-analysis of research on information technology implementation in small business. Journal of Organizational Computing and Electronic Commerce, Mahwah: Lawrence Erlbaum Associates, v. 13, n. 2, p. 91-121, 2003.

TAKAHASHI, T.

Sociedade da Informação no Brasil: Livro Verde. Brasília: Ministério da Ciência e Tecnologia. 2000, p. 203.

TU, Q.

Measuring organizational level IS usage and its impact on manufacturing performance. In: AMERICAS CONFERENCE ON INFORMATION SYSTEMS (AMCIS), 8., 2001. Boston, Proceedings... Boston: Association for Information Systems, p. 2188-2194, 2001. 